\title{
Vol. $40(2013) \mathrm{N}^{\circ} 1$
}

\section{Knowledge Organization}

\section{International Journal}

Devoted to Concept Theory, Classification, Indexing, and Knowledge Representation

\section{CONTENT}

\section{Editorial}

Richard P. Smiraglia.

ISKO 12's Bookshelf-Evolving Intension: An Editorial

\section{Articles}

Birger Hjørland. User-based and Cognitive Approaches to Knowledge Organization :

A Theoretical Analysis of the Research Literature

Mercedes de la Moneda Corrochano, María J. López-Huertas and Evaristo Jiménez Contreras. Spanish Research in Knowledge Organization (2002-2010)

Joseph T. Tennis. Ethos and Ideology of Knowledge : Toward Precepts for an Engaged Knowledge Organization

Maria Luiza de Almeida Campos, Maria Luiza Machado Campos, Alberto M. R. Dávila, Hagar Espanha Gomes, Linair Maria Campos, and Laura de Lira e Oliveira. Sciences Methodological Aspects Applied to Reuse Tools: A Study Based on Genomic in the Domain of Trypanosomatides

\section{Book Review}

Rajendra Kumbhar.

Library Classification Trends in the $21^{\text {st }}$ Century. Witney, UK: Chandos Publishing (Oxford) Ltd.: 2012. ISBN: 1843346605,9781843346609

\section{Classification Issues}

Nancy J. Williamson.

Paradigms and Conceptual Systems in Knowledge Organization, the Eleventh International ISKO Conference, Rome, 2010

\section{Letters to the Editor}

Index to Volume 39 


\section{$\mathrm{KO}$}

\section{KNOWLEDGE ORGANIZATION}

Official Bi-Monthly Journal of the International Society for Knowledge Organization

ISSN $0943-7444$

International Journal devoted to Concept Theory, Classification, Indexing and Knowledge Representation

\section{Contents}

\section{Editorial}

Richard P. Smiraglia.

ISKO 12's Bookshelf-Evolving Intension:

An Editorial 3

\section{Articles}

Birger Hjørland.

User-based and Cognitive Approaches

to Knowledge Organization: A Theoretical

Analysis of the Research Literature

Mercedes de la Moneda Corrochano,

María J. López-Huertas and

Evaristo Jiménez Contreras.

Spanish Research in Knowledge Organization

(2002-2010)

Joseph T. Tennis.

Ethos and Ideology of Knowledge Organization:

Toward Precepts for an Engaged Knowledge

Organization

Maria Luiza de Almeida Campos,

Maria Luiza Machado Campos,

Alberto M. R. Dávila, Hagar Espanha Gomes,

Linair Maria Campos, and Laura de Lira e Oliveira.

Information Sciences Methodological Aspects

Applied to Ontology Reuse Tools: A Study Based

on Genomic Annotations in the Domain of

Trypanosomatides.

\section{Book Review}

Rajendra Kumbhar.

Library Classification Trends in the $21^{\text {st }}$ Century.

Witney, UK: Chandos Publishing (Oxford)

Ltd.: 2012. ISBN: $1843346605,9781843346609$. .62

\section{Classification Issues}

Nancy J. Williamson.

Paradigms and Conceptual Systems in

Knowledge Organization,

the Eleventh International ISKO Conference,

Rome, 2010

\section{Letters to the Editor}

Rick Szostak.

Speaking Truth to Power in Classification:

Response to Fox's Review of My Work;

KO 39:4, 300.

Guohua Xiao.

A Knowledge Classification Model Based on the

Relationship Between Science and Human Needs .77

Index to Volume 39 .79 


\section{KNOWLEDGE ORGANIZATION}

ISSN $0943-7444$

International Journal devoted to Concept Theory, Classification, Indexing and Knowledge Representation

\section{KNOWLEDGE ORGANIZATION}

This journal is the organ of the INTERNATIONAL SOCIETY FOR KNOWLEDGE ORGANIZATION (General Secretariat: Vivien PETRAS, Humboldt-Universität zu Berlin, Institut für Bibliotheks- und Informationswissenschaft, Unter den Linden 6, 10099 Berlin, Germany. E-mail: secr@isko.org.

\section{Editors}

Dr. Richard P. SMIRAGLIA (Editor-in-Chief), School of Information Studies, University of Wisconsin, Milwaukee, Northwest Quad Building B, 2025 E Newport St., Milwaukee, WI 53211 USA. E-mail: smiragli@uwm.edu

Dr. Joseph T. TENNIS (Book Review Editor), The Information School of the University of Washington, Box 352840, Mary Gates Hall Ste 370, Seattle WA 98195-2840 USA.

E-mail: jtennis@u.washington.edu

Dr. Nancy WILLIAMSON (Classification Research News Editor), Faculty of Information Studies, University of Toronto, 140 St. George Street, Toronto, Ontario M5S 3G6 Canada.

E-mail: william@fis.utoronto.ca

David J. BLOOM (Editorial Assistant), School of Information Studies, University of Wisconsin, Milwaukee, Northwest Quad Building B, 2025 E Newport St., Milwaukee, WI 53211 USA.

Melodie Joy FOX (Editorial Assistant), School of Information Studies, University of Wisconsin, Milwaukee, Northwest Quad Building B, 2025 E Newport St., Milwaukee, WI 53211 USA.

Daniel Martínez Ávila (Editorial Assistant), Department of Library and Information Science, University Carlos III of Madrid, C/Madrid 12628903 Getafe - Madrid, Spain.

E-mail:dmartine@bib.uc3m.es

\section{Editors Emerita}

Dr. Hope A. OLSON, School of Information Studies, 522 Bolton Hall, University of Wisconsin-Milwaukee, Milwaukee, Northwest Quad Building B, 2025 E Newport St., Milwaukee, WI 53211 USA.E-mail: holson@uwm.edu

Dr. Clare BEGHTOL, Faculty of Information Studies, University of Toronto, 140 St. George Street, Toronto, Ontario M5S 3G6, Canada. E-mail: clare.beghtol@utoronto.ca

Ingetraut DAHLBERG, Am Hirtenberg 13, 64732 Bad König, Germany. E-mail: IDahlberg@t-online.de

\section{Editorial Board}

Dr. Jonathan FURNER, Graduate School of Education \& Information Studies, University of California, Los Angeles, 300 Young Dr. N, Mailbox 951520, Los Angeles, CA 90095-1520, USA. E-mail: furner@gseis.ucla.edu

Prof. Jesús GASCÓN GARCÍA, Facultat de Biblioteconomia i Documentació, Universitat de Barcelona, C. Melcior de Palau, 140, 08014 Barcelona, Spain. E-mail: gascon@ub.edu

Claudio GNOLI, University of Pavia, Mathematics Department Library, via Ferrata 1, I-27100 Pavia, Italy. E-mail: gnoli@aib.it
Dr. Rebecca GREEN, Assistant Editor, Dewey Decimal Classification, Dewey Editorial Office, Library of Congress, Decimal Classification Division, 101 Independence Ave., S.E., Washington, DC 20540-4330, USA.E-mail: greenre@oclc.org

Dr. José Augusto Chaves GUIMARÃES, Departamento de Ciência da Infromação, Universidade Estadual Paulista-UNESP, Av. Hygino Muzzi Filho 737, 17525-900 Marília SP Brazil.

E-mail: guima@marilia.unesp.br

Dr. Birger HJØRLAND, Royal School of Library and Information Science, Copenhagen Denmark. E-mail: bh@iva.dk

Dr. Barbara H. KWASNIK, School of Information Studies, Syracuse University, Syracuse, NY 13244 USA.

E-mail: bkwasnik@syr.edu

Dr. María J. LÓPEZ-HUERTAS. Universidad de Granada, Facultad de Biblioteconomía y Documentación, Campus Universitario de Cartuja, Biblioteca del Colegio Máximo de Cartuja, 18071 Granada, Spain.E-mail: mjlopez@ugr.es

Dr. Kathryn LA BARRE, The Graduate School of Library and Information Science, University of Illinois at Urbana-Champaign, 501 E. Daniel Street, MC-493, Champaign, IL 61820-6211 USA. E-mail klabarre@illinois.edu

Dr. Marianne LYKKE, e-Learning Lab, Center for User-driven Innovation, Learning and Design, Department of Communication, Aalborg University, Kroghstraede 1, room 2.023 Denmark 9220 Aalborg OE. E-mail: mlykke@hum.aau.dk

Dr. Ia MCILWAINE (Literature Editor), Research Fellow. School of Library, Archive \& Information Studies, University College London, Gower Street, London WC1E 6BT U.K.

E-mail: i.mcilwaine@ucl.ac.uk

Dr. Jens-Erik MAI, Royal School of Library and Information Science, Copenhagen Denmark. E-mail: jem@iva.dk

Dr. Widad MUSTAFA el HADI, Université Charles de Gaulle Lille 3, URF IDIST, Domaine du Pont de Bois, Villeneuve d'Ascq 59653, France. E-mail: widad.mustafa@free.fr

H. Peter OHLY, Prinzenstr. 179, D-53175 Bonn, Germany.

E-mail: president@isko.org

Dr. K. S. RAGHAVAN, DRTC, Indian Statistical Institute, Bangalore 560 059, India. E-mail: raghavan@isibang.ac.in

Dr. M. P. SATIJA, Guru Nanak Dev University, School of Library and Information Science, Amritsar-143 005, India.

E-mail: satija_mp@yahoo.com

Dr. Aida SLAVIC, UDC Consortium, PO Box 90407, 2509 LK The Hague, The Netherlands. E-mail: aida.slavic@udcc.org

Dr. Dagobert SOERGEL, Department of Library and Information Studies, Graduate School of Education, University at Buffalo, 534 Baldy Hall, Buffalo, NY 14260-1020.

E-mail: dsoergel@buffalo.edu

Dr. Renato R. SOUZA, Applied Mathematics School, Getulio Vargas Foundation, Praia de Botafogo, 190, 3o andar, Rio de Janeiro, RJ, 22250-900, Brazil. E-mail: renato.souza@fgv.br

Dr. Maja ŽUMER, Faculty of Arts, University of Ljubljana, Askerceva 2, Ljubljana 1000 Slovenia, E-mail: maja.zumer@ff.uni-lj.si 